\title{
Potential Diagnostic Value Of Combining
}

\section{Inflammatory Cell Ratios With Carcinoembryonic Antigen For Colorectal Cancer}

This article was published in the following Dove Press journal:

Cancer Management and Research

\author{
Xinxin $\mathrm{Li}^{1}{ }^{1} *$ \\ Dongming Guol,* \\ Lingyu $\mathrm{Chu}^{2}$ \\ Yiteng Huang ${ }^{3}$ \\ Feiran Zhang' \\ Wei $\mathrm{Li}^{1}$ \\ Juntian Chen'
}

'Department of Gastrointestinal Surgery, The First Affiliated Hospital of Shantou University Medical College, Shantou, People's Republic of China; ${ }^{2}$ Department of Biochemistry and Molecular Biology, Shantou University Medical College, Shantou, People's Republic of China; ${ }^{3}$ Department of Health Care Center, The First Affiliated Hospital of Shantou University Medical College, Shantou, People's Republic of China

*These authors contributed equally to this work
Correspondence: Juntian Chen Department of Gastrointestinal Surgery, The First Affiliated Hospital of Shantou University Medical College, 57 Changping Road, Shantou 5I504I, Guangdong Province, People's Republic of China $\mathrm{Tel} / \mathrm{Fax}+8675488905256$

Email 13809846668@I63.com
Purpose: To evaluate the diagnostic value of combining the neutrophil-lymphocyte ratio (NLR), platelet-lymphocyte ratio (PLR) or lymphocyte-monocyte ratio (LMR) with carcinoembryonic antigen (CEA) in patients with colorectal cancer (CRC).

Patients and methods: The diagnostic performance of inflammatory makers and CEA was evaluated in cohort 1 (664 patients with CRC, 336 patients with colorectal polyps and 664 healthy controls) and validated in cohort 2 ( 87 patients with CRC and 87 healthy controls) by using receiver operating characteristic curve analysis.

Results: In cohort 1, the NLR, PLR and CEA levels were significantly higher, while the LMR was markedly lower in patients with CRC than in healthy controls. The PLR and LMR were significantly associated with invasion depth and lymph node metastasis. Moreover, significant differences in the PLR and LMR were observed between patients with stage I/II CRC and healthy or polyp controls and those with stage III/IV CRC. Using the NLR, PLR or LMR with CEA resulted in a significantly larger area under the curve (AUC) than any of them used alone. Combining the PLR and LMR with CEA exhibited the best diagnostic value for $\mathrm{CRC}(\mathrm{AUC}=0.892$ ). The AUCs of this combination were 0.864 and 0.783 for distinguishing stage I/II CRC from healthy and polyp controls, respectively. When we used the same cut-off values to assess the diagnostic ability of these markers in cohort 2 , similar results were observed, and the PLR, LMR and CEA combination also showed the highest accuracy (AUC=0.936).

Conclusion: Combining inflammatory cell ratios with CEA could improve the diagnostic efficacy for CRC patients. The combination of the PLR and LMR with CEA might be a valuable indicator in the early detection and monitoring of CRC patients.

Keywords: colorectal cancer, neutrophil-lymphocyte ratio, platelet-lymphocyte ratio, lymphocyte-monocyte ratio, carcinoembryonic antigen, diagnosis

\section{Introduction}

Colorectal cancer (CRC) is one of the most common malignant tumors around the world and ranks third in morbidity and mortality. ${ }^{1}$ Over 1.8 million new colorectal cancer cases and 881,000 deaths were estimated to occur in 2018 worldwide. ${ }^{2}$ In China, an estimated 376,300 newly diagnosed cases and 191,000 CRC-related deaths occurred in $2015 .^{3}$ Early CRC is asymptomatic, and most cases are at advanced stages when they are diagnosed. The 5-year survival rate can reach $90 \%$ for patients with early-stage disease, while it drops to $11.7 \%$ in metastatic CRC patients. ${ }^{4}$ Early and accurate diagnosis is important for the treatment and prognosis of CRC patients. However, 
current diagnostic methods for early CRC are still not effective. Fecal occult blood tests are widely used to screen CRC, but they have low sensitivity and specificity. Colonoscopy is considered to be the gold standard for the detection of earlystage CRC. However, it is limited from being a regular screening method because of its expensiveness, invasiveness and risk during the examination. ${ }^{5}$ Carcinoembryonic antigen (CEA) is regarded as the most important and common serological biomarker for the detection and monitoring of CRC but has insufficient sensitivity and specificity. ${ }^{6}$ Hence, it is urgent to explore non-invasive and valuable diagnostic biomarkers for the early diagnosis of CRC.

Recently, a large number of studies have shown that inflammation is associated with the development and progression of various cancers, including colorectal cancer. ${ }^{7-9}$ Inflammation promotes carcinogenesis, and inflammatory indices reflect the systemic inflammatory response. ${ }^{10}$ Peripheral blood inflammatory parameters are easily measured and widely detected for patients with $\mathrm{CRC}$ in clinical practice. At the same time, there is growing evidence that circulating blood inflammatory markers, such as the neutrophil-lymphocyte ratio (NLR), the platelet-lymphocyte ratio (PLR), the lymphocyte-monocyte ratio (LMR), C-reactive protein, haptoglobin and albumin, are correlated with the diagnosis or prognosis of CRC. ${ }^{11-15}$ The NLR and PLR have been reportedly found to be associated with invasion depth and tumor stage and can be used for the early diagnosis and outcome prediction of CRC patients. ${ }^{16}$ The LMR has also been reported to be related to tumor differentiation grade and metastasis and can thus provide valuable information about diagnosis and prognosis in colorectal cancer. ${ }^{17}$ Recent studies indicated that the combination of inflammatory parameters and tumor markers has better diagnostic value for malignant tumors. ${ }^{18-20}$ However, little is known about the diagnostic efficacy of combining inflammatory biomarkers with tumor markers in CRC. Thus, the aim of this study was to evaluate the single diagnostic accuracy of the preoperative NLR, PLR, LMR and CEA levels in CRC patients and investigate whether the combination of these biomarkers could improve diagnostic efficacy.

\section{Materials And Methods Study Samples}

In this study, cohort 1 included 664 patients with CRC, 336 patients with colorectal polyps and 664 healthy examinees matched by sex and age. In cohort 2, there were 87 patients with CRC and 87 sex- and age-matched healthy examinees. The participants in cohort 1 and cohort 2 were selected from the First Affiliated Hospital of Shantou University Medical College from July 2014 to June 2018 and from July 2018 to December 2018, respectively. The inclusion criteria for CRC patients were as follows: 1) patients who did not have infections, hematologic diseases, tissue disorders, coronary artery or cerebrovascular diseases, other bowel diseases or other types of cancers; 2) patients who did not receive any preoperative anticancer treatments, such as chemotherapy or radiotherapy; 3) patients who were histologically confirmed to have colorectal adenocarcinoma by two pathologists after operation; and 4) patients with complete data. The tumor stages were classified according to the 8th edition of the AJCC cancer staging system. ${ }^{21}$ In our study, stage I/II was defined as early stage, while stage III/IV was defined as advanced stage. The enrolled patients with colorectal polyps and healthy examinees were used as polyp controls and healthy controls (HC), respectively. All included healthy examinees had no clinical evidence of any diseases according to their health check at the hospital.

Patient identities were anonymized before analysis. The requirement for informed consent was waived because this was a retrospective study. The protocol was approved by the Ethics Committee of the First Affiliated Hospital of Shantou University Medical College, China.

\section{Methods}

The pathological features and blood tests were obtained from postoperative pathology and preoperative laboratory examination, respectively. A $2 \mathrm{~mL}$ EDTA-anticoagulated blood sample was collected to detect the counts of inflammatory cells using a Beckman LH 750 analyzer (Beckman Coulter, Brea, CA). The NLR and PLR were calculated by dividing the absolute number of neutrophils or platelets, respectively, by the absolute number of lymphocytes. The LMR was calculated by dividing the absolute lymphocyte count by the absolute monocyte count. A $2 \mathrm{~mL}$ serum sample was obtained to examine the level of CEA using the chemiluminescence method with a Beckman DxI 800 machine (Beckman Coulter, Brea, CA). The average time of sampling from CRC patients was 3 days before the date of surgery. All fasting venous blood samples were collected from the enrolled cases and controls between 6:00 am and 9:00 am and analyzed within $1 \mathrm{hr}$ after venipuncture. Internal quality control and external quality assessment were applied to validate the quality and comparability of the test results. 


\section{Statistical Analysis}

The statistical analyses were performed using SPSS 24.0 (Chicago, IL, USA), GraphPad Prism 7 (San Diego, CA, USA) and MedCalc 15.2.2 software (Mariakerke, Belgium). Continuous variables are presented as the mean \pm standard deviation or median (quartile). The normality of the calculated variables was assessed by using the Kolmogorov-Smirnov test. Student's $t$-test and the Mann-Whitney $U$-test were used for normally and nonnormally distributed data, respectively. The Kruskal-Wallis test was applied for comparisons among three groups. The chi-square test was used to compare categorical data. The combined diagnostic ability of the inflammatory cell ratios and CEA was assessed using binary logistic analysis to calculate the overall predictive probability and was further evaluated using receiver operating characteristic (ROC) curve analysis. ROC curve analysis was performed by MedCalc version 15.2.2 to identify the diagnostic value of the NLR, PLR, LMR and CEA level, which was determined by calculating the cut-off value, area under the ROC curve (AUC), sensitivity, specificity and Youden index. $P<0.05$ was considered statistically significant.

\section{Results}

\section{Baseline Characteristics}

The clinicopathological characteristics of the patients in cohort 1 and cohort 2 are summarized in Table 1. Age and sex did not differ significantly between CRC patients and healthy controls in either cohort 1 or 2 . In cohort 1 , compared with healthy controls, CRC patients had significantly higher levels of circulating neutrophils, platelets, monocytes, NLRs, PLRs and CEA as well as lower levels of lymphocytes and LMRs. Similar results were observed in cohort 2.

\section{Associations Between Clinical Characteristics And Pretreatment NLR, PLR, LMR And CEA Levels}

As Table 2 shows, in cohort 1, the level of the NLR was significantly related to invasion depth, but no significant differences were observed in lymphatic metastasis, distant metastasis and TNM stage. The PLR level showed a significant correlation with invasion depth, lymphatic metastasis and TNM stage. However, no significant relation was identified between the PLR and distant metastasis. Decreased LMR levels and increased CEA levels were significantly associated with the depth of tumor invasion, lymph node metastasis, distant metastasis and TNM stage.
In addition, compared with 349 age- and sex-matched healthy controls and 336 polyp controls, the levels of the NLR, the PLR and CEA were significantly increased, whereas the LMR was obviously decreased in early-stage CRC patients in cohort 1 (Table 3 and Figure 1).

\section{Diagnostic Values Of The NLR, PLR, LMR And CEA Level Used Alone And In Combination For Patients With CRC}

In cohort 1, ROC curve analyses suggested that the optimum cut-off values for the NLR, PLR, LMR and CEA level were $1.81,128.03,4.61$ and 3.30 , respectively. The AUC values of the NLR, PLR, LMR and CEA level were 0.723 (95\% CI: 0.698-0.747), 0.779 (95\% CI: 0.756 $0.802), 0.800$ (95\% CI: $0.778-0.821)$ and 0.792 (95\% CI: 0.769-0.813), respectively. Combining the NLR with CEA (AUC: $0.840,95 \%$ CI: 0.819-0.859) demonstrated higher diagnostic value than using the NLR or CEA alone $(P<0.001)$. Similar results $(P<0.001)$ were observed for CEA in conjunction with the PLR (AUC: $0.873,95 \% \mathrm{CI}$ : 0.854-0.891) or the LMR (AUC: $0.874,95 \%$ CI: 0.855 0.891). Moreover, using any two of these inflammatory biomarkers combined with CEA produced a larger AUC than using a single inflammatory marker with CEA. The combination of the PLR, the LMR and CEA had the largest AUC (AUC: 0.892, 95\% CI: 0.874-0.908) of all types of combined biomarkers (Table 4 and Figure 2). When we used the same cut-off values to evaluate the diagnostic accuracy of these markers in cohort 2, similar results were observed, and the combination of the PLR, the LMR and CEA also exhibited the best diagnostic value (AUC=0.936).

\section{Diagnostic Efficacy Of The PLR, LMR And CEA Combination For Early-Stage CRC Patients}

Our results indicated that the combination of the PLR, the LMR and CEA was the best combination for the diagnosis of CRC. Therefore, the diagnostic efficacy of this combination was further evaluated in early CRC. As Figure 3 shows, in cohort 1, the AUC values of the PLR, LMR and CEA combination were 0.864 (95\% CI: $0.836-$ 0.889 ) and 0.783 (95\% CI: $0.750-0.813$ ) for diagnosis of early-stage CRC patients from healthy and polyp controls, respectively. 
Table I Clinicopathological Features And Laboratory Parameters In CRC Patients And Healthy Controls

\begin{tabular}{|c|c|c|c|c|}
\hline \multirow[t]{2}{*}{ Variable } & \multicolumn{2}{|l|}{ Cohort I } & \multicolumn{2}{|l|}{ Cohort 2} \\
\hline & CRC Patients $(n=664)$ & Healthy Controls $(n=664)$ & CRC Patients $(n=87)$ & Healthy Controls $(n=87)$ \\
\hline Age (years) & $64(56-7 I)$ & $64(56-7 I)$ & $63(54-70)$ & $63(54-70)$ \\
\hline \multicolumn{5}{|l|}{ Sex } \\
\hline Male & 369 (55.6\%) & $369(55.6 \%)$ & $54(62.1 \%)$ & $54(62.1 \%)$ \\
\hline Female & $295(44.4 \%)$ & 295 (44.4\%) & $33(37.9 \%)$ & $33(37.9 \%)$ \\
\hline \multicolumn{5}{|l|}{ Location } \\
\hline Colon & $385(58.0 \%)$ & & $59(67.8 \%)$ & \\
\hline Rectum & $279(42.0 \%)$ & & $28(32.2 \%)$ & \\
\hline \multicolumn{5}{|l|}{ Grade } \\
\hline Low & 285 (42.9\%) & & 39 (44.8\%) & \\
\hline Moderate & $272(41.0 \%)$ & & $35(40.2 \%)$ & \\
\hline High & $107(16.1 \%)$ & & $13(15.0 \%)$ & \\
\hline \multicolumn{5}{|l|}{ Lymphatic invasion } \\
\hline Yes & $387(58.3 \%)$ & & $54(62.1 \%)$ & \\
\hline No & $277(41.7 \%)$ & & $33(37.9 \%)$ & \\
\hline \multicolumn{5}{|l|}{ Vascular invasion } \\
\hline Yes & $349(52.6 \%)$ & & 47 (54.0\%) & \\
\hline No & $315(47.4 \%)$ & & $40(46.0 \%)$ & \\
\hline \multicolumn{5}{|l|}{ TNM stage } \\
\hline I & 78 (1 I.7\%) & & $6(6.9 \%)$ & \\
\hline II & $27 \mid(40.8 \%)$ & & $30(34.5 \%)$ & \\
\hline III & $248(37.4 \%)$ & & 39 (44.8\%) & \\
\hline VI & $67(10.1 \%)$ & & $12(13.8 \%)$ & \\
\hline \multicolumn{5}{|l|}{ Invasion depth } \\
\hline TI & $28(4.2 \%)$ & & $2(2.3 \%)$ & \\
\hline $\mathrm{T} 2$ & $58(8.7 \%)$ & & $6(6.9 \%)$ & \\
\hline $\mathrm{T} 3$ & 124 (I8.7\%) & & $18(20.7 \%)$ & \\
\hline T4 & $454(68.4 \%)$ & & 69 (70.1\%) & \\
\hline \multicolumn{5}{|l|}{ Lymphatic metastasis } \\
\hline No & $360(54.2 \%)$ & & $38(43.7 \%)$ & \\
\hline NI & 197 (29.7\%) & & $35(40.2 \%)$ & \\
\hline N2 & $107(16.1 \%)$ & & $14(16.1 \%)$ & \\
\hline \multicolumn{5}{|l|}{ Distant metastasis } \\
\hline Mo & 597 (89.9\%) & & $75(86.2 \%)$ & \\
\hline MI & $67(10.1 \%)$ & & $12(13.8 \%)$ & \\
\hline Neutrophils $\left(10^{9} / \mathrm{L}\right)^{\mathrm{a}, \mathrm{b}}$ & $4.27(3.23-5.75)$ & $3.44(2.80-4.12)$ & $4.42(3.10-5.46)$ & $3.58(2.95-4.14)$ \\
\hline Lymphocytes $\left(10^{9} / \mathrm{L}\right)^{\mathrm{a}, \mathrm{b}}$ & $1.85(1.43-2.31)$ & $2.14(1.76-2.64)$ & $1.78(1.45-2.15)$ & $2.24(1.98-2.75)$ \\
\hline Platelets $\left(10^{9} / \mathrm{L}\right)^{\mathrm{a}, \mathrm{b}}$ & $256.00(214.00-321.75)$ & $203.00(171.00-234.00)$ & $278.00(220.00-363.00)$ & $208.00(185.00-238.00)$ \\
\hline Monocytes $\left(10^{9} / \mathrm{L}\right)^{\mathrm{a}, \mathrm{b}}$ & $0.53(0.40-0.67)$ & $0.39(0.30-0.48)$ & $0.53(0.42-0.66)$ & $0.42(0.35-0.50)$ \\
\hline$N L R^{a, b}$ & $2.23(I .65-3.4 I)$ & $1.58(1.26-2.0 \mathrm{I})$ & $2.46(1.68-3.28)$ & $1.53(1.30-1.86)$ \\
\hline$P_{L R} R^{a, b}$ & |38.6| (105.37-197.30) & $92.50(75.03-115.03)$ & $165.90(122.11-209.36)$ & $94.68(78.15-112.50)$ \\
\hline$L M R^{a, b}$ & $3.52(2.58-4.77)$ & $5.67(4.52-7.00)$ & $3.43(2.79-4.05)$ & $5.24(4.57-6.87)$ \\
\hline $\operatorname{CEA}(\mathrm{ng} / \mathrm{mL})^{\mathrm{a}, \mathrm{b}}$ & $4.26(2.21-11.37)$ & $1.80(1.00-2.78)$ & $4.06(2.17-10.93)$ & $1.60(1.00-2.40)$ \\
\hline
\end{tabular}

Notes: ${ }^{\mathrm{a}} P<0.05$ : CRC patients vs healthy controls in cohort I; ${ }^{\mathrm{b}} \mathrm{P}<0.05$ : CRC patients vs healthy controls in cohort 2.

Abbreviations: CRC, colorectal cancer; HC, healthy control; NLR, neutrophil-lymphocyte ratio; PLR, platelet-lymphocyte ratio; LMR, lymphocyte-monocyte ratio; CEA, carcinoembryonic antigen. 
Table 2 Associations Of Preoperative NLR, PLR And CEA Levels With TNM Stage In CRC Patients In Cohort I

\begin{tabular}{|c|c|c|c|c|c|c|c|c|c|}
\hline Groups & $\mathbf{N}$ & NLR & $P$ & PLR & $P$ & LMR & $P$ & CEA & $P$ \\
\hline $\begin{array}{l}\text { T stage } \\
\text { T1+T2 } \\
\text { T3+T4 }\end{array}$ & $\begin{array}{l}86 \\
578\end{array}$ & $\begin{array}{l}1.86(1.51-2.28) \\
2.33(1.68-3.58)\end{array}$ & $<0.001$ & $\begin{array}{l}\text { I I } 5.58(96.90-139.24) \\
\text { | } 42.37(106.82-207.40)\end{array}$ & $<0.001$ & $\begin{array}{l}4.36(3.4 I-5.34) \\
3.43(2.43-4.6 I)\end{array}$ & $<0.001$ & $\begin{array}{l}2.42(1.64-3.88) \\
4.82(2.33-13.18)\end{array}$ & $<0.001$ \\
\hline $\begin{array}{l}\mathrm{N} \text { stage } \\
\quad \mathrm{N} 0 \\
\mathrm{~N} 1+\mathrm{N} 2\end{array}$ & $\begin{array}{l}360 \\
304\end{array}$ & $\begin{array}{l}2.17(1.66-3.26) \\
2.32(1.64-3.57)\end{array}$ & 0.278 & $\begin{array}{l}\text { | } 32.34(|03.08-| 78.5 \mid) \\
\mid 43.92(\mid 06.75-209.18)\end{array}$ & 0.031 & $\begin{array}{l}3.63(2.75-4.88) \\
3.39(2.40-4.60)\end{array}$ & 0.036 & $\begin{array}{l}3.72(2.13-8.77) \\
4.95(2.39-13.64)\end{array}$ & 0.008 \\
\hline $\begin{array}{c}\text { M stage } \\
\text { Mo } \\
\text { MI }\end{array}$ & $\begin{array}{l}597 \\
67\end{array}$ & $\begin{array}{l}2.21(1.64-3.39) \\
2.52(1.85-3.64)\end{array}$ & 0.088 & $\begin{array}{l}138.30(\mid 03.91-197.08) \\
|4| .98(\mid 15.79-203.25)\end{array}$ & 0.235 & $\begin{array}{l}3.58(2.63-4.8 I) \\
3.04(2.25-4.22)\end{array}$ & 0.017 & $\begin{array}{l}3.72(2.13-8.74) \\
12.90(6.39-102.85)\end{array}$ & $<0.001$ \\
\hline $\begin{array}{l}\text { Stage } \\
\qquad \begin{array}{l}|+| \mid \\
I I I+\mid V\end{array}\end{array}$ & $\begin{array}{l}349 \\
315\end{array}$ & $\begin{array}{l}2.14(1.66-3.23) \\
2.36(1.64-3.57)\end{array}$ & 0.186 & $\begin{array}{l}132.12(102.92-176.62) \\
\mid 44.02(|07.2|-209.30)\end{array}$ & 0.019 & $\begin{array}{l}3.66(2.79-4.89) \\
3.37(2.38-4.59)\end{array}$ & 0.017 & $\begin{array}{l}3.57(2.12-8.07) \\
5.28(2.4 \mid-14.50)\end{array}$ & 0.001 \\
\hline
\end{tabular}

Abbreviations: CRC, colorectal cancer; NLR, neutrophil-lymphocyte ratio; PLR, platelet-lymphocyte ratio; LMR, lymphocyte-monocyte ratio; CEA, carcinoembryonic antigen.

Table 3 Comparison Of Clinical Features And Laboratory Parameters Among The Three Groups In Cohort I

\begin{tabular}{|c|c|c|c|c|}
\hline Variable & Early-Stage CRC Patients & Healthy Controls & Polyp Controls & $P$ \\
\hline $\mathrm{n}$ & 349 & 349 & 336 & \\
\hline Age (years) & $65(58-72)$ & $65(58-72)$ & $63(58-70)$ & 0.260 \\
\hline Sex (male, \%) & $196(56.2 \%)$ & $196(56.2 \%)$ & $194(57.7 \%)$ & 0.891 \\
\hline Neutrophils $\left(10^{9} / L\right)^{\mathrm{a}, \mathrm{b}}$ & $4.25(3.35-5.58)$ & $3.35(2.77-4.12)$ & $3.59(2.92-4.30)$ & $<0.001$ \\
\hline Lymphocytes $\left(10^{9} / \mathrm{L}\right)^{\mathrm{a}, \mathrm{b}}$ & $1.87(1.50-2.39)$ & $2.18(1.75-2.68)$ & $2.10(1.68-2.55)$ & $<0.001$ \\
\hline Platelets $\left(10^{9} / \mathrm{L}\right)^{\mathrm{a}, \mathrm{b}, \mathrm{c}}$ & $249.00(211.00-313.50)$ & $202.00(170.00-233.00)$ & $221.00(186.25-258.75)$ & $<0.001$ \\
\hline Monocytes $\left(10^{9} / \mathrm{L}\right)^{\mathrm{a}, \mathrm{b}, \mathrm{c}}$ & $0.52(0.40-0.67)$ & $0.38(0.31-0.48)$ & $0.47(0.39-0.57)$ & $<0.001$ \\
\hline$N L R^{a, b, c}$ & $2.14(1.66-3.23)$ & $1.57(1.22-1.96)$ & $1.69(1.32-2.25)$ & $<0.001$ \\
\hline$P L^{a, b, c}$ & 132.12 (102.92-176.62) & $90.70(74.53-115.95)$ & $105.24(82.80-138.50)$ & $<0.001$ \\
\hline $\operatorname{LMR}^{\mathrm{a}, \mathrm{b}, \mathrm{c}}$ & $3.66(2.79-4.89)$ & $5.74(4.6 \mathrm{I}-7.09)$ & $4.51(3.55-5.85)$ & $<0.001$ \\
\hline $\operatorname{CEA}(n g / m L)^{a, b}$ & $3.57(2.12-8.07)$ & $1.90(1.10-2.70)$ & $1.75(1.16-2.97)$ & $<0.001$ \\
\hline
\end{tabular}

Notes: ${ }^{2}$ Adjusted $P<0.05$ : CRC patients with early stages vs healthy controls; ${ }^{b} A d j u s t e d ~ P<0.05$ : CRC patients with early stages vs polyp controls; ${ }^{\mathrm{C}} \mathrm{Adjusted} P<0.05$ : polyp controls vs healthy controls.

Abbreviations: CRC, colorectal cancer; NLR, neutrophil-lymphocyte ratio; PLR, platelet-lymphocyte ratio; LMR, lymphocyte-monocyte ratio; CEA, carcinoembryonic antigen.

\section{Discussion}

Chronic inflammation plays an important role in promoting tumor initiation, invasion and metastasis. ${ }^{22}$ Patients with inflammatory bowel disease are at an increased risk of developing CRC. ${ }^{23}$ The NLR, PLR and LMR are the most commonly used indicators of systemic inflammatory response and have been reportedly associated with poorer outcomes in CRC patients. ${ }^{12,24,25}$ Kilincalp et al analyzed 144 CRC patients and found that the preoperative NLR and PLR were significantly higher in the CRC patients than in 143 age-matched and sexmatched healthy participants, which suggested that the NLR and PLR might be used as potential biomarkers for the detection and monitoring of CRC. ${ }^{16}$ Our study indicated the same result that patients with CRC had elevated NLRs and PLRs compared to those in healthy controls. In addition, our results showed that the level of the LMR was significantly lower in CRC patients than in healthy controls. Although growing evidence has revealed that inflammatory markers seem to be promising screening factors for CRC patients, inflammatory indices are associated with various diseases and are easily influenced by a variety of other factors. Thus, the use of a single inflammatory marker for the detection of CRC may produce deviations. To reduce the interference of other factors, combining markers of other mechanisms may provide compensation and improve the diagnostic performance. Recent studies have reported that combining inflammatory biomarkers with tumor markers could increase the diagnostic efficacy of malignant tumors. ${ }^{18-20}$ 
A

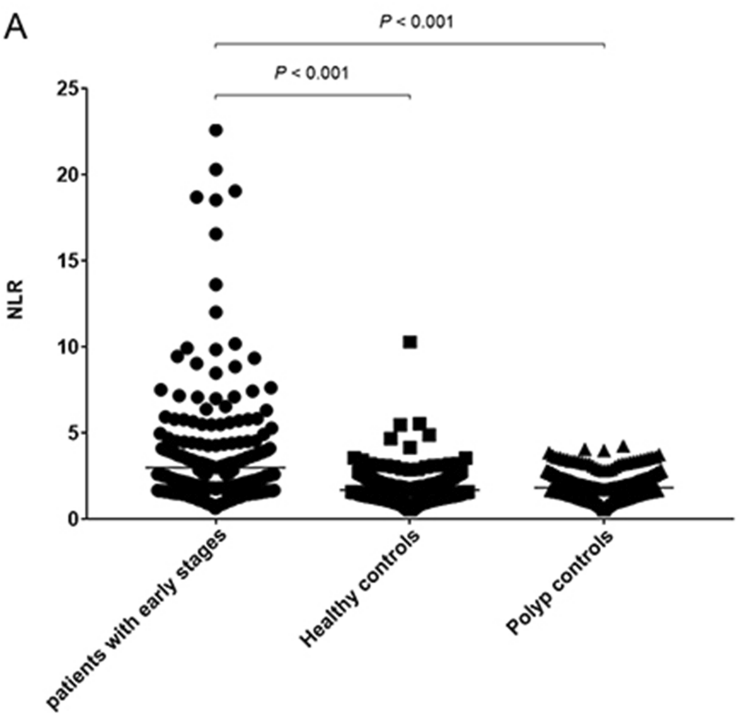

C

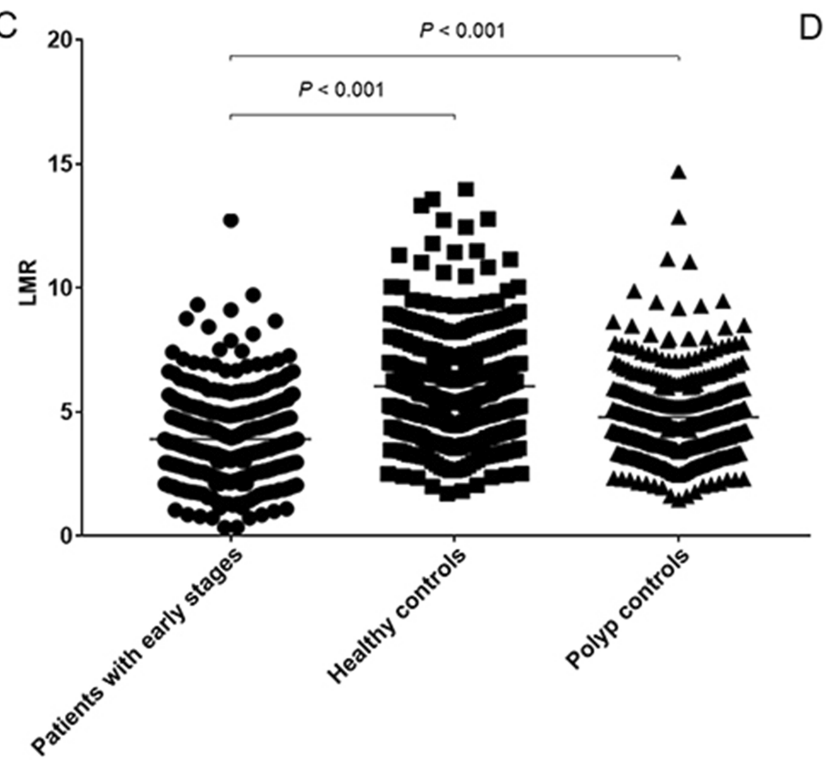

B

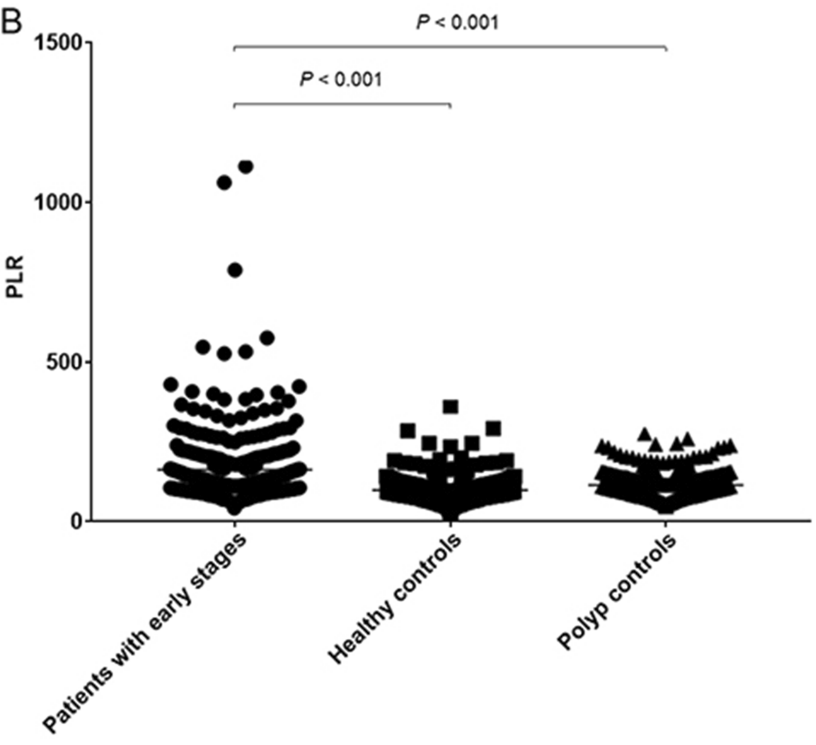

D

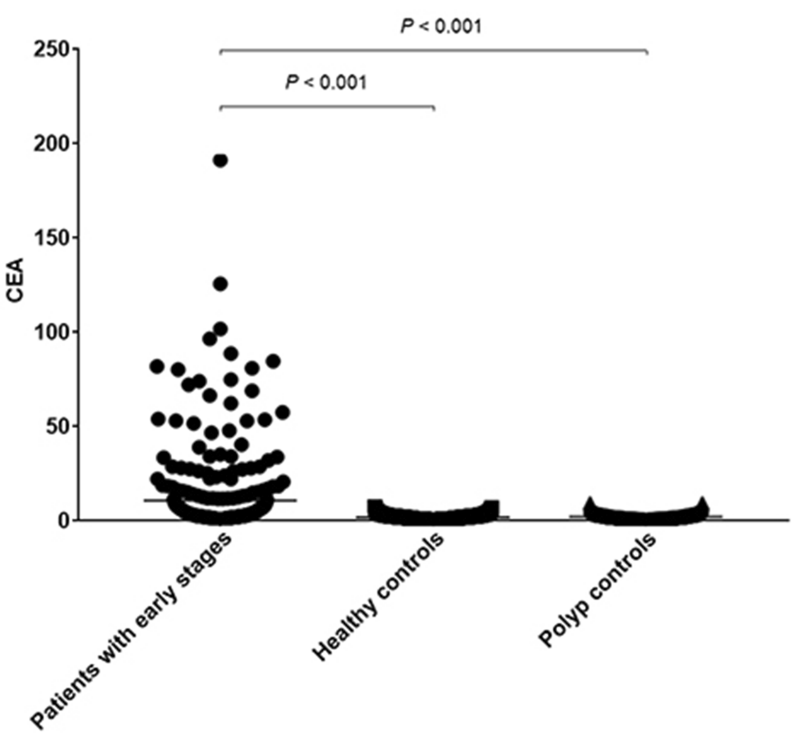

Figure I The different levels of inflammatory cell ratios and CEA among CRC patients with early stages, healthy controls and polyp controls in cohort I. (A) The NLR in the three groups. (B) The PLR in the three groups. (C) The LMR in the three groups. (D) The CEA level in the three groups.

Abbreviations: CEA, carcinoembryonic antigen; CRC, colorectal cancer; NLR, neutrophil-lymphocyte ratio; PLR, platelet-lymphocyte ratio; LMR, lymphocyte-monocyte ratio.

CEA, a serum glycoprotein, is secreted by tumors located in hollow organs and is the most widely used marker for CRC, but its diagnostic accuracy remains limited due to its unsatisfactory sensitivity and specificity. ${ }^{6}$ Our results demonstrated that using inflammatory cell ratios with CEA was superior to using any of them alone for the diagnosis of CRC. We found that combining the NLR and PLR with CEA produced larger AUC values than using the NLR, the PLR or CEA alone, which was consistent with the findings of a previous report. ${ }^{18}$ One of our interesting findings was that the combination of the
PLR, the LMR and CEA had significantly higher values than single or other combined biomarkers in the diagnosis of CRC. To our knowledge, our study is the first to investigate the diagnostic role of the PLR and LMR combined with CEA in patients with CRC. Another interesting finding was that CRC patients with early stages exhibited significantly elevated PLRs and decreased LMRs compared to healthy and polyp controls, suggesting that the combination of the PLR, the LMR and CEA could effectively distinguish early CRC from healthy and polyp controls. Moreover, our study demonstrated that both the PLR 
Table 4 Diagnostic Efficiency Of The NLR, PLR And CEA Level Alone And In Combination For Patients With CRC

\begin{tabular}{|c|c|c|c|c|c|c|c|c|c|c|}
\hline Variable & $\begin{array}{l}\text { Cut-Off } \\
\text { Value }\end{array}$ & AUC & $95 \% \mathrm{Cl}$ & Sensitivity (\%) & Specificity (\%) & PPV (\%) & NPV (\%) & +LR & -LR & Youden Index \\
\hline \multicolumn{11}{|l|}{ Cohort I } \\
\hline$N_{L} R^{a}$ & 1.81 & 0.723 & $0.698-0.747$ & 69.13 & 65.21 & 66.52 & 67.87 & 1.99 & 0.47 & 0.3434 \\
\hline $\operatorname{PLR}^{\mathrm{a}}$ & 128.03 & 0.779 & $0.756-0.802$ & 57.23 & 85.39 & 79.66 & 66.63 & 3.92 & 0.50 & 0.4262 \\
\hline $\mathrm{LMR}^{\mathrm{a}}$ & 4.61 & 0.800 & $0.778-0.821$ & 72.89 & 73.80 & 73.56 & 73.13 & 2.78 & 0.37 & 0.4669 \\
\hline $\mathrm{CEA}^{\mathrm{a}}$ & 3.30 & 0.792 & $0.769-0.813$ & 58.28 & 85.54 & 80.12 & 67.22 & 4.03 & 0.49 & 0.4383 \\
\hline $\mathrm{NLR}+\mathrm{CEA}^{\mathrm{a}}$ & 0.459 & 0.840 & $0.819-0.859$ & 69.28 & 83.28 & 80.56 & 73.05 & 4.14 & 0.37 & 0.5256 \\
\hline $\mathrm{PLR}+\mathrm{CEA}^{\mathrm{a}}$ & 0.567 & 0.873 & $0.854-0.891$ & 66.72 & 91.72 & 88.96 & 73.37 & 8.06 & 0.36 & 0.5843 \\
\hline $\mathrm{LMR}+\mathrm{CEA}^{\mathrm{a}}$ & 0.564 & 0.874 & $0.855-0.891$ & 71.84 & 87.05 & 84.72 & 75.56 & 5.55 & 0.32 & 0.5889 \\
\hline $\mathrm{NLR}+\mathrm{PLR}+\mathrm{CEA}^{\mathrm{a}}$ & 0.553 & 0.874 & $0.855-0.891$ & 67.92 & 91.27 & 88.61 & 73.99 & 7.78 & 0.35 & 0.5919 \\
\hline $\mathrm{NLR}+\mathrm{LMR}+\mathrm{CEA}^{\mathrm{a}}$ & 0.530 & 0.874 & $0.855-0.891$ & 73.49 & 85.09 & 83.13 & 76.25 & 4.93 & 0.31 & 0.5858 \\
\hline$P L R+L M R+C E A$ & 0.485 & 0.892 & $0.874-0.908$ & 76.81 & 85.69 & 84.30 & 78.70 & 5.37 & 0.27 & 0.6250 \\
\hline \multicolumn{11}{|l|}{ Cohort 2} \\
\hline NLR & 1.81 & 0.787 & $0.718-0.845$ & 71.26 & 70.11 & 70.45 & 70.93 & 2.38 & 0.41 & 0.4137 \\
\hline PLR & 128.03 & 0.850 & $0.788-0.900$ & 68.97 & 88.51 & 85.71 & 74.04 & 6.00 & 0.35 & 0.5748 \\
\hline LMR & 4.61 & 0.865 & $0.805-0.912$ & 89.66 & 74.71 & 78.00 & 87.84 & 3.55 & 0.14 & 0.6437 \\
\hline CEA & 3.30 & 0.816 & $0.75 I-0.87 \mid$ & 55.17 & 90.80 & 85.71 & 66.95 & 6.00 & 0.49 & 0.4597 \\
\hline $\mathrm{NLR}+\mathrm{CEA}$ & 0.459 & 0.903 & $0.849-0.943$ & 80.46 & 85.06 & 84.34 & 81.32 & 5.38 & 0.23 & 0.6552 \\
\hline $\mathrm{PLR}+\mathrm{CEA}$ & 0.567 & 0.928 & $0.879-0.962$ & 79.31 & 90.80 & 89.61 & 81.44 & 8.63 & 0.23 & 0.7011 \\
\hline $\mathrm{LMR}+\mathrm{CEA}$ & 0.564 & 0.907 & $0.854-0.946$ & 74.71 & 89.66 & 87.84 & 78.00 & 7.22 & 0.28 & 0.6437 \\
\hline$N L R+P L R+C E A$ & 0.553 & 0.930 & $0.882-0.963$ & 80.46 & 90.80 & 89.74 & 82.29 & 8.75 & 0.22 & 0.7126 \\
\hline $\mathrm{NLR}+\mathrm{LMR}+\mathrm{CEA}$ & 0.530 & 0.912 & $0.860-0.950$ & 78.16 & 88.51 & 87.18 & 80.21 & 6.80 & 0.25 & 0.6667 \\
\hline PLR+LMR+CEA & 0.485 & 0.936 & $0.889-0.968$ & 81.61 & 89.66 & 88.75 & 82.98 & 7.89 & 0.21 & 0.7127 \\
\hline
\end{tabular}

Notes: ${ }^{a} P<0.05$ : AUC for the biomarker vs combined PLR, LMR with CEA in cohort I.

Abbreviations: AUC, area under the receiver operating characteristic curve; $95 \% \mathrm{Cl}, 95 \%$ confidential interval; PPV, positive predictive value; NPV, negative predictive value; +LR, positive likelihood ratio; -LR, negative likelihood ratio; CRC, colorectal cancer; NLR, neutrophil-lymphocyte ratio; PLR, platelet-lymphocyte ratio; LMR, lymphocyte-monocyte ratio; CEA, carcinoembryonic antigen.

and LMR were significantly different between patients with early stages and those with advanced stages, which is similar to the findings of previous reports. ${ }^{11,26}$ Significant differences in the PLR and LMR were observed between patients with early stages and healthy controls, benign controls or those with advanced stages, suggesting that the two markers may contribute to tumor initiation and progression. Moreover, the PLR and LMR are routinely detected not only in the pretreatment assessment of patients with CRC but also in the physical examination of healthy people. Therefore, the combination of the PLR and LMR with CEA might be a potential marker in the early diagnosis of CRC.

Our results can be explained by the following mechanisms. Cancer-related inflammation, the interaction between cancer and the systemic inflammatory response, is an essential process in malignant tumors. Platelets are conducive to tumor growth, angiogenesis and metastasis by producing growth and angiogenic factors, such as vascular endothelial growth factor and basic fibroblast growth factor. ${ }^{27}$ Additionally, platelets provide favorable conditions for cancer cells by aggregating around them, including immune surveillance escape, shear stress protection, prosurvival signals, adhesion to the endothelium and extravasation. ${ }^{28}$ Interactively, cancer cells, through their oncogenic transformation, can release many platelet agonists, such as adenosine diphosphate, thrombin, thromboxane and microparticles, which participate in platelet activation and contribute to thrombocythemia in cancer patients. ${ }^{28,29}$ In fact, according to a previous study, CRC patients with high preoperative platelet counts exhibit poor survival. ${ }^{30}$ Lymphocytes, which play a crucial role in cytotoxic cell death, can inhibit the proliferation and metastatic spread of tumor cells. ${ }^{31}$ The spontaneous apoptosis of peripheral $\mathrm{T}$ cells is frequently observed in cancer patients and may be stimulated by programmed cell death-1, lymphocyte activation gene-3 and caspase-3 activity. ${ }^{32,33}$ A decreased peripheral lymphocyte count may indicate the hypofunction of immune surveillance and inferior lymphocyte-medicated immune response to tumor progression. Peripheral monocytes are recruited to tumors and differentiate into macrophages by a wide variety of growth factors and chemokines produced by tumor cells, 

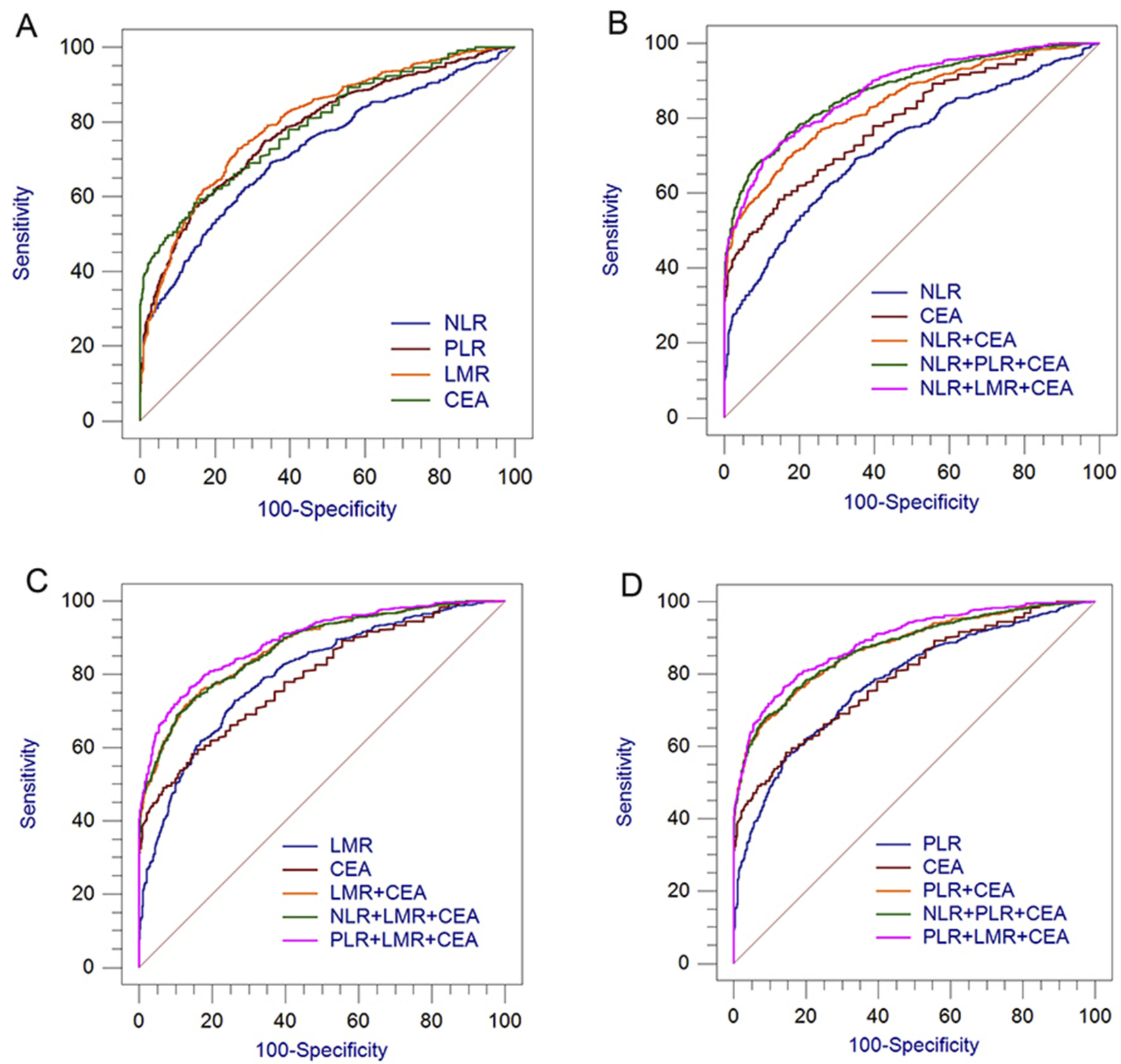

Figure 2 Diagnostic values of single and combined inflammatory cell ratios with CEA for CRC from healthy controls in cohort I. (A) NLR, PLR, LMR and CEA for CRC. (B) NLR combined with other biomarkers for CRC. (C) PLR combined with other biomarkers for CRC. (D) LMR combined with other biomarkers for CRC.

Abbreviations: CEA, carcinoembryonic antigen; CRC, colorectal cancer; NLR, neutrophil-lymphocyte ratio; PLR, platelet-lymphocyte ratio; LMR, lymphocyte-monocyte ratio.

such as colony-stimulating factor 1 , macrophage-stimulating protein, transforming growth factor- $\beta 1$ and macrophage inflammatory protein-1 $\alpha^{34,35}$ Macrophages can initiate tumorigenesis and promote tumor progression by producing high levels of reactive oxygen, fibroblast growth factor and so on. ${ }^{34,35}$ Recent studies have reported that lymphopenia and monocytosis are inversely correlated with poor prognosis in patients with CRC. ${ }^{36}$

According to the above mechanisms, the NLR, PLR and CEA levels seem to increase with tumor progression, whereas the LMR is likely to decrease with tumor progression. A significant difference in the PLR, LMR and CEA levels between early CRC and advanced CRC patients was observed in our study, which is in agreement with these mechanisms. Therefore, this combination may also be a simple but effective marker for identifying whether the tumor is progressing in the monitoring of CRC.

This study has several limitations. First, it was a singlecenter and retrospective study, which might result in 

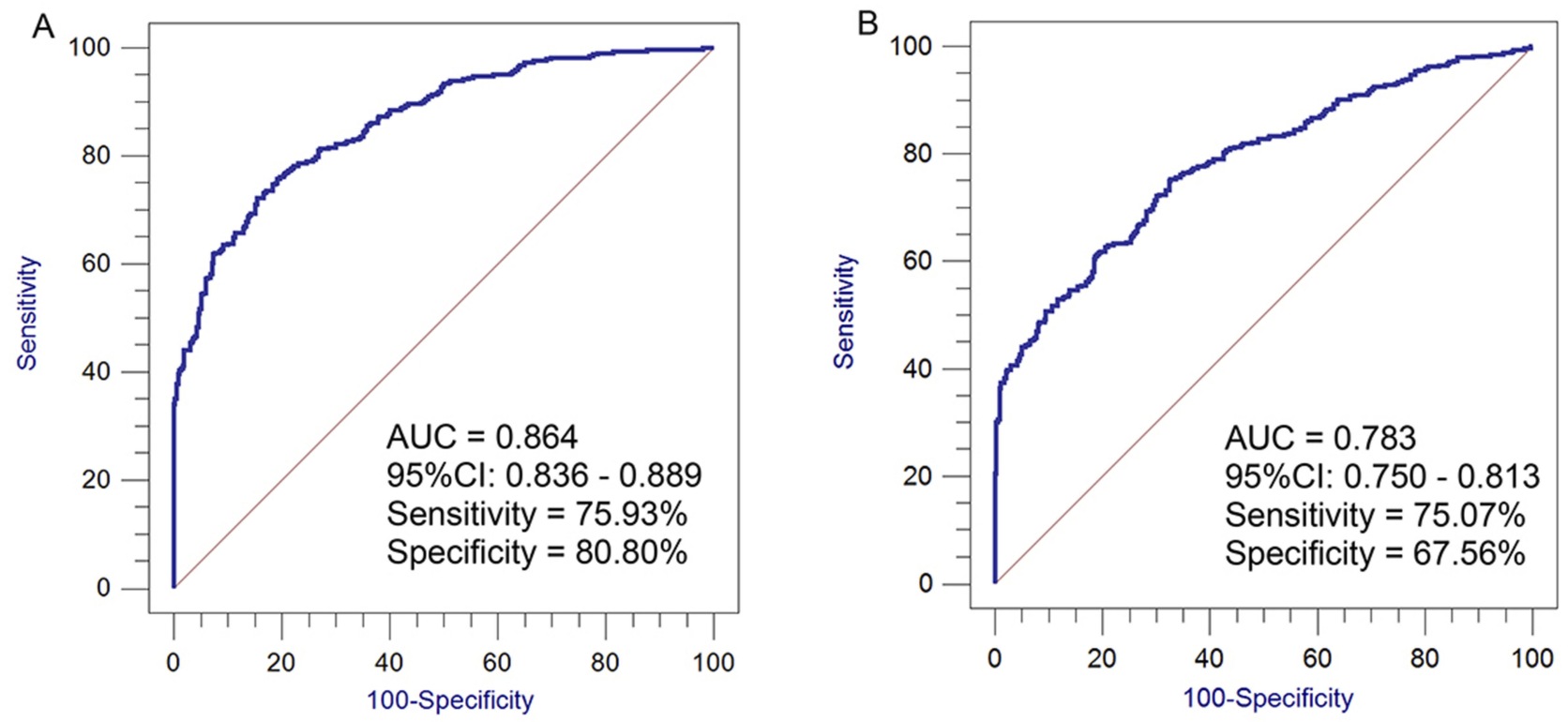

Figure 3 Combining the PLR and LMR with CEA for distinguishing early-stage CRC patients from healthy controls and polyp controls in cohort I. (A) The PLR and LMR combined with CEA for distinguishing early-stage CRC patients from age- and sex-matched healthy controls. (B) The PLR and LMR combined with CEA for distinguishing early-stage CRC patients from polyp controls.

Abbreviations: CRC, colorectal cancer; NLR, neutrophil-lymphocyte ratio; PLR, platelet-lymphocyte ratio; LMR, lymphocyte-monocyte ratio; CEA, carcinoembryonic antigen.

selection bias when collecting information on participants. Second, the method was highly standardized, and the enrolled individuals were almost all from Guangdong province; thus, the results may not be applicable to other regions. Third, the population of external validation was small; therefore, more external cohorts are needed to confirm these results.

\section{Conclusion}

In conclusion, the NLR, PLR or LMR in combination with CEA can improve the diagnostic efficacy of CRC. The combination of the PLR, LMR and CEA level might be a useful indicator in the early detection and monitoring of $\mathrm{CRC}$ and has better diagnostic value than other combined markers.

\section{Acknowledgments}

We appreciate the support received from the Department of Clinical Medicine Laboratory Center, the First Affiliated Hospital of Shantou University Medical College, Shantou, China.

\section{Disclosure}

The authors report no conflicts of interest in this work.

\section{References}

1. Siegel RL, Miller KD, Jemal A. Cancer statistics, 2018. CA Cancer J Clin. 2018;68(1):7-30. doi:10.3322/caac.21442

2. Bray F, Ferlay J, Soerjomataram I, Siegel RL, Torre LA, Jemal A. Global cancer statistics 2018: GLOBOCAN estimates of incidence and mortality worldwide for 36 cancers in 185 countries. CA Cancer J Clin. 2018. doi:10.3322/caac.21492

3. Chen W, Zheng R, Baade PD, et al. Cancer statistics in China, 2015. CA Cancer J Clin. 2016;66(2):115-132. doi:10.3322/caac.21338

4. Brenner H, Kloor M, Pox CP. Colorectal cancer. Lancet. 2014;383 (9927):1490-1502. doi:10.1016/S0140-6736(13)61649-9

5. Lieberman D. Colorectal cancer screening with colonoscopy. JAMA Intern Med. 2016;176(7):903-904. doi:10.1001/jamainternmed.2016. 1333

6. McKeown E, Nelson DW, Johnson EK, et al. Current approaches and challenges for monitoring treatment response in colon and rectal cancer. J Cancer. 2014;5(1):31-43. doi:10.7150/jca.7987

7. Coussens LM, Werb Z. Inflammation and cancer. Nature. 2002;420 (6917):860-867. doi:10.1038/nature01322

8. Kuraishy A, Karin M, Grivennikov SI. Tumor promotion via injuryand death-induced inflammation. Immunity. 2011;35(4):467-477. doi:10.1016/j.immuni.2011.09.006

9. Cho Y, Lee J, Oh J, et al. Inflammatory dietary pattern, IL-17F genetic variant, and the risk of colorectal cancer. Nutrients. 2018;10 (6):724. doi:10.3390/nu10060724

10. Garcia-Anguita A, Kakourou A, Tsilidis KK. Biomarkers of inflammation and immune function and risk of colorectal cancer. Curr Colorectal Cancer Rep. 2015;11(5):250-258. doi:10.1007/s11888015-0282-5

11. Jia J, Zheng X, Chen Y, et al. Stage-dependent changes of preoperative neutrophil to lymphocyte ratio and platelet to lymphocyte ratio in colorectal cancer. Tumor Biol. 2015;36(12):9319-9325. doi:10.1007/ s13277-015-3667-9 
12. Tan D, Fu Y, Tong W, Li F. Prognostic significance of lymphocyte to monocyte ratio in colorectal cancer: a meta-analysis. Int J Surg. 2018;55:128-138. doi:10.1016/j.ijsu.2018.05.030

13. Holm M, Saraswat M, Joenväärä S, Ristimäki A, Haglund C, Renkonen R. Colorectal cancer patients with different C-reactive protein levels and 5-year survival times can be differentiated with quantitative serum proteomics. PLoS One. 2018;13(4):e0195354. doi:10.1371/journal.pone.0195354

14. Sun L, Hu S, Yu L, et al. Serum haptoglobin as a novel molecular biomarker predicting colorectal cancer hepatic metastasis. Int $J$ Cancer. 2016;138(11):2724-2731. doi:10.1002/ijc.v138.11

15. González-Trejo S, Carrillo JF, Carmona-Herrera DD, et al. Baseline serum albumin and other common clinical markers are prognostic factors in colorectal carcinoma. Medicine. 2017;96(15):e6610. doi:10.1097/MD.0000000000006610

16. Kilincalp S, Çoban Ş, Akinci H, et al. Neutrophil/lymphocyte ratio, platelet/lymphocyte ratio, and mean platelet volume as potential biomarkers for early detection and monitoring of colorectal adenocarcinoma. Eur J Cancer Prev. 2015;24(4):328-333. doi:10.1097/ CEJ.0000000000000092

17. Kostakis ID, Vaiopoulos AG, Garoufalia Z, et al. What can preoperative blood tests tell us about colorectal cancer? J Buon. 2018;23 (7):84-95.

18. Peng H-X, Yang L, He B-S, et al. Combination of preoperative NLR, PLR and CEA could increase the diagnostic efficacy for I-III stage CRC. J Clin Lab Anal. 2017;31(5):e22075. doi:10.1002/jcla.2017.31. issue-5

19. Wu Y, Jiang M, Qin Y, Lin F, Lai M. Single and combined use of neutrophil-lymphocyte ratio, platelet-lymphocyte ratio and carcinoembryonic antigen in diagnosing gastric cancer. Clin Chim Acta. 2018;481:20-24. doi:10.1016/j.cca.2018.02.027

20. Liu XF, Zhou LY, Wei ZH, et al. The diagnostic role of circulating inflammation-based biomarker in gallbladder carcinoma. Biomark Med. 2018;12(10):1095-1103. doi:10.2217/bmm-2018-0049

21. Weiser MR. AJCC 8th edition: colorectal cancer. Ann Surg Oncol. 2018;25(6):1454-1455. doi:10.1245/s10434-018-6462-1

22. Mantovani A, Allavena P, Sica A, Balkwill F. Cancer-related inflammation. Nature. 2008;454(7203):436-444. doi:10.1038/nature07205

23. Ullman TA, Itzkowitz SH. Intestinal inflammation and cancer. Gastroenterology. 2011;140(6):1807-1816. doi:10.1053/j.gastro.2011. 01.057

24. Li MX, Liu XM, Zhang XF, et al. Prognostic role of neutrophil-tolymphocyte ratio in colorectal cancer: a systematic review and metaanalysis. Int J Cancer. 2014;134(10):2403-2413. doi:10.1002/ijc.28536
25. Min GT, Wang YH, Yao N, et al. The prognostic role of pretreatment platelet-to-lymphocyte ratio as predictors in patients with colorectal cancer: a meta-analysis. Biomark Med. 2017;11(1):87-97. doi:10.22 17/bmm-2016-0181

26. Chan JCY, Chan DL, Diakos CI, et al. The lymphocyte-to-monocyte ratio is a superior predictor of overall survival in comparison to established biomarkers of resectable colorectal cancer. Ann Surg. 2017;265(3):539-546. doi:10.1097/SLA.0000000000001743

27. Schlesinger M. Role of platelets and platelet receptors in cancer metastasis. J Hematol Oncol. 2018;11:1. doi:10.1186/s13045-0180669-2

28. Plantureux L, Crescence L, Dignat-George F, Panicot-Dubois L, Dubois C. Effects of platelets on cancer progression. Thromb Res. 2018;164:S40-S47. doi:10.1016/j.thromres.2018.01.035

29. Mezouar S, Mege D, Darbousset R, et al. Involvement of plateletderived microparticles in tumor progression and thrombosis. Semin Oncol. 2014;41(3):346-358. doi:10.1053/j.seminoncol.2014.04.010

30. Monreal M, Fernandez-Llamazares J, Pinol M, et al. Platelet count and survival in patients with colorectal cancer-a preliminary study. Thromb Haemost. 1998;79(5):916-918. doi:10.1055/s-0037-1615093

31. Ferradini L, Miescher S, Stoeck M, et al. Cytotoxic potential despite impaired activation pathways in T lymphocytes infiltrating nasopharyngeal carcinoma. Int J Cancer. 1991;47(3):362-370. doi:10.1002/ (ISSN)1097-0215

32. Matsuzaki J, Gnjatic S, Mhawech-Fauceglia P, et al. Tumor-infiltrating NY-ESO-1-specific CD8+ T cells are negatively regulated by LAG-3 and PD-1 in human ovarian cancer. Proc National Acad Sci. 2010;107(17):7875-7880. doi:10.1073/pnas.1003345107

33. Takahashi A, Kono K, Amemiya H, Iizuka H, Fujii H, Matsumoto Y. Elevated caspase- 3 activity in peripheral blood $\mathrm{T}$ cells coexists with increased degree of T-cell apoptosis and down-regulation of TCR zeta molecules in patients with gastric cancer. Clin Cancer Res. 2001;7(1):74-80.

34. Pollard JW. Tumour-educated macrophages promote tumour progression and metastasis. Nat Rev Cancer. 2004;4(1):71-78. doi:10.1038/ nrc 1256

35. Condeelis J, Pollard JW. Macrophages: obligate partners for tumor cell migration, invasion, and metastasis. Cell. 2006;124(2):263-266. doi:10.1016/j.cell.2006.01.007

36. Tanio A, Saito H, Uejima C, et al. A prognostic index for colorectal cancer based on preoperative absolute lymphocyte, monocyte, and neutrophil counts. Surg Today. 2019;49(3):245-253. doi:10.1007/ s00595-018-1728-6

\section{Publish your work in this journal}

Cancer Management and Research is an international, peer-reviewed open access journal focusing on cancer research and the optimal use of preventative and integrated treatment interventions to achieve improved outcomes, enhanced survival and quality of life for the cancer patient.
The manuscript management system is completely online and includes a very quick and fair peer-review system, which is all easy to use. Visit http://www.dovepress.com/testimonials.php to read real quotes from published authors. 